\title{
ILANG EKSPLORASYON \\ SA PAG-AARAL NG KULTURANG POPULAR SA FILIPINAS
}

\author{
Louie Jon A. Sánchez \\ Ateneo de Manila University \\ Isanchez@ateneo.edu
}

\begin{abstract}
Pacing Caroline Hau (2002), this essay explores the historical and intellectual specificities of Cultural Studies and the study of Popular Culture in the Philippines, and situates them in the author's recent study of the cultural history of the teleserye, the Filipino TV soap opera. While the essay assumes that much of the key ideas and paradigms of Western Cultural Studies have been adopted in the country, the ethos of the local field may also be traced back to the works of patriots José Rizal and Isabelo delos Reyes. The projects of the two have been continued by intellectuals and scholars who emerged during the 1960s to the 1970 and formulated more postcolonial approaches to the study of Philippine culture. Moreover, the essay comments on the importance and role of interdisciplinarity in the emergence of Cultural Studies and the study of Popular Culture in the Philippines.
\end{abstract}

\section{Keywords}

cultural studies; popular culture; interdisciplinarity; José Rizal; Isabelo delos Reyes; teleserye

\section{About the Author}

Louie Jon A. Sánchez teaches writing, literature, popular culture, and television studies at the Department of English, Ateneo de Manila University. He recently earned a PhD in Literature from De La Salle University, Manila, where he presented a dissertation on the cultural history of the teleserye, the Filipino TV soap opera. He is the author of At Sa Tahanan ng Alabok (Poetry in Filipino, U of Santo Tomas P House), Kung Saan sa Katawan (Poetry in Filipino, UST), Pagkahaba-haba Man ng Prusisyon (Essays, U of the Philippines P), Aralin at Siyasat (Criticism, UST), and the forthcoming Abangán: Mga Pambungad na Resepsiyon sa Kultura ng Teleserye sa Filipinas (Criticism, Ateneo de Naga U P). 
Sa mahalagang sanaysay na "The 'Cultural' and 'Linguistic' Turns in Philippine Scholarship," tinukoy ni Caroline Hau (2002) ang tinawag niyang "pihit pa-wika/ pihit pa-kultura" bílang yugto ng pagpasok ng mga susing kabaguhang intelektwal noong masalimuot at masigalot na mga dekada 6o hanggang 70 sa bansa (37). Samantalang nakaangkla ang talakay sa kaniyang nagkukrus na interes sa mga larang ng panitikan (partikular sa nobela) at kasaysayan, malinaw na naitanghal niya ang pagiging litaw ng pihit sa pangmalawakang mga larang ng humanidades at aralíng panlipunan, kung saan, aniya, maigting at walang maliw ang "debates over the proper boundaries," kahit pa, wika rin niya, "the disciplines themselves have never been rigorous formations to begin with" (36).

Papaano nga bang binago ng mga nasabing kabaguhang intelektwal ang mga larang, lalo na ang pag-aaral ng panitikan sa Filipinas? Itinanghal niya sa panimula ang naging malaong hinaing hinggil sa malaganap na paggamit ng "academic jargon" drawn from disciplines outside the literary field"; sa animo'y pagliliwaliw ng teoryang pampanitikan sa iba't ibang larang na napag-uugnayan nito ng mga hinuha; at manapa, sa "intellectual cannibalization" o "borrowing of terms and concepts... as a digression from the true vocation of criticism or, worse, a threat to the hard-earned autonomy of literary studies as a viable discipline" (36).

Lalang ang mga jargon, ang "bagong wikang" nabanggit, ng "emergence of literary theory" in the wake of the shift from a loosely defined "structuralist" movement in the "human sciences" to an even more loosely defined post-structuralism in the humanities" (49). Sinasabing nagrurok ang pos-estrukturalismo sa America, matapos ang inilalarawang "misreading" kay Jacques Derrida (49). Nagsilbing tagapagsulong ng mga kaisipan ni Derrida, na "nagtatag" sa "dekonstruksiyon," ang mga kagawaran ng panitikan mula sa mga pamantasan sa America, at lalo na maging sa Inglatera, kung saan higit na naging matalab ang mga panibagong pagbásang Marxismo sa pag-aaral sa araw-araw na búhay at kultura ng taumbayan.

Pagsapit ng dekada 8o, at paglipas ng halos 20 taóng pagpapalawig ng diskurso, isang baguntao ang lilitaw na kakatawan sa mga lawas ng kaalamang hatid ng pagkakayanig ni Derrida sa sistema ng pakahulugan at karunungan: ang "critical theory." Ang teoryang kritikal na ito ang babakasing magulang ng kinikilala na natin ngayon na "Cultural Studies" o yaong nais kong tawagin na Aralíng Pangkultura.

Hinggil sa Aralíng Pangkultura, nakasandig ang ating pagpapakahulugan dito bílang isang pagdulog, pagpapalawak ng pag-unawa sa kultura mismo bílang malawak na kagawian ng madla, at pagtibag sa mga halagahang pangkultura na mapagsantabi sa yaong di makapapasa sa "matataas" na pamantayan, at wika nga nina Julie Rivkin at Michael Ryan (2004), ay pagsusuri rito bílang "means of domination, of assuring the rule of one class or group over another, and means 
of resistance to such domination," suma total, "a way of articulating oppositional points of view to those in dominance" (1213).

Kayâ kilala ito bílang larang na tumititig-at tumititig nang walang pagpapaumanhin—sa tinatawag na pangmadlang kultura o mass culture bílang mapagpakahulugan at hindi lámang "tool(s) of domination, a way for capitalism to offer ephemeral gratification to people condemned to lives of work" (1213). Paksa ng aralín ang kabuuang kulturang popular, mula sa mga palathalaang mapagpalaganap, pangmadlang komunikasyon, mga kulturang nasasalaysayan at subkultura, at marami pang maaaring kabasahan ng pagpapakahulugang masasabing tinatangkilik o kagawian ng mga "nasa ibaba."

Ngunit ang nabanggit ay isa lámang sa mga sityo ng Aralíng Pangkultura kung babalikan ang mga pinagbukalan nito, halimbawa, sa Inglatera noong dekada 6o. Ang Aralíng Pangkultura ng mga Ingles, lalo pa't sa pagkakatatag ng tanyag na Centre for Contemporary Cultural Studies sa Birmingham University, isang "decisive organizational instance" (6) na sinasabi ni Chris Barker (200o), ay bumubuo sa mga pormasyong diskursibo na kung pagbabatayan ang isa sa mga tagapagtatag nitong si Stuart Hall ay "a cluster (or formation) of ideas, images and practices, which provide ways of talking about, forms of knowledge and conduct associated with a particular topic, social activity or institutional site in society" (sinipi kay Baker 6).

Sa pangkabuuan, kinikilala ang Aralíng Pangkultura bílang nagtataglay ng mga sumusunod na katangian, sang-ayon sa pagpapakahulugan dito ni Tony Bennett, isa pa nitong tagapagsulong: (1) interdisplinaridad, na kaisipang bumabaklas sa mga hanggahan ng mga larangan sa ngalan ng pagsusuri sa "relations of culture and power"; (2) pagbaling sa lahat ng mga "practices, institutions and systems of classification," na nagkakaloob at nagtatakda para sa mamamayan ng mga "particular values, beliefs, competencies, routines of life and habitual forms of conduct"; (3) paglalantad sa mga ugnayan ng kapangyarihang umiiral sa kasarian, lahi, uri, kolonyalismo, atbp., upang makapaghayin ng "ways of thinking about culture and power that can be utilized by agents in the pursuit of change"; at pagabot sa mga "social and political movements, workers in cultural institutions, and cultural management," sa kabila ng pagiging nakapook sa akademya at mataas na edukasyon (sinipi kay Baker 7).

Sa Filipinas, talagang malaki ang utang na loob nito sa larang ng panitikan, sapagkat di tulad sa Kanluran, kung saan babád ito sa pagsusuring pangmidya, ititindig ito bandáng dekada 70 ng mga paham na Filipinong hinutok ng aralíng pampanitikan (Tolentino at Devilles 2), matapos masagap ang mga katuruan nito, madalas, sa kani-kanilang mga pag-aaral sa Kanluran. Magsisilbi itong gambala 
at pagyanig sa umiiral na pagkahaling ng mga intelektwal sa mapagtakdang New American Criticism dahil sa mapagpalayang pananaw nitong tumitibag sa mga nakaugaliang pagpapahalagang artistiko lalo sa panitikan.

Sa ganang akin, masasabi ring ang pagkakasagap sa Aralíng Pangkultura-sa pamamagitan man ng pag-aaral sa Kanluran o pagbása sa mga susing tekstong nakaabot sa bansa, di naglaon-ang lalong nagpatatag sa naunang intelektwal na mithiing makapagtindig ng malalim na paghinuha hinggil sa mas malawakang kulturang Filipinong madalas na naisasantabi dahil sa ating danas at edukasyong kolonyal at madaling naisasantabi dahil na rin umiiral ito sa pang-araw-araw.

Bílang susog sa tangka sa kasaysayang intelektwal ni Hau, nais kong imungkahi ang pagturing din sa pihit pa-kultura bilang yugto ng muling "pagkágísing," lalo pa't sa nasabing panahong masalimuot at masigalot rin nagsimula ang pagtatanong muli hinggil sa kaakuhang Filipino.

Nagagabayan ang papel na ito ng mga sumusunod na tanong sa pagtuklas nito: Papaanong umusbong o umunlad ang "pihit pa-kultura" sa Filipinas? Sinosinong naging tagapanguna ng pihit na ito at ano-ano ang kanilang ambag? May mga natatangi bang usapin na tumatalakay hinggil sa mga sitwasyon o realidad na Filipino, na may kaugnayan, halimbawa, sa pag-aaral ng teleserye na aking ginawa kamakailan? Papaanong naiiba o sumasalok ang mga kombersasyong ito mula sa mga susing teorista sa larang ng Aralíng Pangkultura?

May bagahe mang Kanluranin, dinulutan pa rin ng pihit pa-kultura ang mga palaisip sa bansa ng isang bagong wika at pananaw upang sikaping balikán at tunghan ang sariling kalinangan na nawawaglit o naliligtaan. Kung sinasabing dinestrungka ng pihit pa-kultura ang mga saligan ng Kanluraning kaisipan, animo'y dinestrungka naman nito, sa pagdating sa Filipinas, ang tanikalang nagpapanatili sa intelektwal na tradisyon sa pagsalig sa nasabing tinalikuran nang mga kaisipan.

Nararapat ipagpauna na ang sanaysay na ito ay isang tangka sa pagbuo sa kritikal na balangkas ng aking naging pag-aaral sa kasaysayan ng pag-angkop, pag-unlad, at pagbago sa soap opera sa Filipinas sa loob ng mahigit 80 taon. Inilulugar nito ang aking pinagmumulan bílang mananaliksik na nagsisikap hinuhain ang isang tekstong pangkulturang malaong kalát (unwieldy, massive), at marahil dahil na nga rin doon ay tumanggap ng pang-iismir at kakulangan sa pagbaling mula sa mga mapagmataas sa taga-akademya sa mahabang panahon.

Dalawang bagay ang nagtulak sa akin na sulátin ito. Pangunahin na rito ang paglulugar-manapa'y "pagbubuntot" - sa aking pag-aaral sa nagpapatúloy na mga paghinuha sa praktika at teorya ng kulturang popular. Naisip kong maisasagawa 
ito kung tutuklasin ko, kahit sa isang panimulang paraan, ang isa pang mahalagang kasaysayan, ang kasaysayan ng pag-aaral at pagpapahalaga sa kulturang popular sa bansa-siyang pangalawa't matalik na kaugnay na layon ng una. Kayâ personal at intelektwal ang gawaing ito sa mula't mula, isang paliwanag sa sariling sa hulíy iniisip kong maaaring maibahagi lalo sa mga sangkot din sa mga parehong gawain, upang higit na mapalawig at mapalalim ang mga talakay.

\section{PAGBABALIK KINA JOSÉ RIZAL AT ISABELO DELOS REYES}

Hindi sapat na tukuyin lámang ang pihit pa-kultura sa Filipinas bílang pihit pateorya, samantalang napakalaki nga ng ambag ng teorya, lalo pa sa paglawak ng Aralíng Pangkultura sa loob at labas ng Filipinas, at sa pagpapalaganap nito bílang larang. Ang "pagteteorya" hinggil sa Filipinong kaakuhan ay matagal nang tinutupad, at kailangang bakasin ito sa mga panahong kolonyal, kung kailan umusbong ang pagsasalaysay, kung baga, ng masasabing mga ideyang pambansa at makabansa. Ito ang totoong nagpapa-iba rito bílang isang nakapagsasariling "kasaysayan ng karunungan."

Sa aking pananaw, tumulong lámang ang pagdating ng teorya sa lalong pagusad ng nagpapatúloy na proseso ng "pagharaya" sa ating komunidad, wika nga ni Benedict Anderson. Pangunahin sa mga kaisipang teoretiko na naiisip kong talagang nagtulak sa ating mga lokal na teorista ay ang mismong mapagbuwag na diwa ng pos-estrukturalismo na higit na naglantad sa mga mapanikil na estruktura ng kaisipang namana natin mula sa mananakop.

Iginiit nito ang matagal nang hininagap, halimbawa nina José Rizal at Isabelo Delos Reyes, na ang pagbubuo at pagsasalaysay sa kalinangan ng mga katutubo ang siyang pinakamabuting tugon sa pagmamaliit na kanilang nakukuha mula sa mapagmataas na sumasakop. Internalisado nang maituturing ang ating pook bílang barbaro at pagano at kailangan ng pagliligtas ng Kanluran. Kayâ marahil, sa kabila ng lubhang kahirapan ng pos-estrukturalismo, at kahit pa napakatindi ng pagkasarado ng ating mga nakagisnang pananampalataya, naghatid ito ng bagong kislap-diwa at wari'y ipinagunita ang matagal nang sinasabi sa atin ng mga unang lumaban sa kolonya.

Umusbong at umunlad ang pihit pa-kultura bílang pagpapatúloy, sa isang bagong wika, ng pagsasaysay at pagsasalaysay ng ating kaakuhan, bagaman isang bagay na pinagdududahan sa pos-estrukturalismo ang pananalig sa mga esensiyal at paghagilap pa sa "ubod" ng kalinangan. Sa pagbásang ito, kung gayon, nag-iilang diwa ang teoretikong sandali sapagkat hindi na lámang bumabaling sa yanig na 
dulot ng pagkakalantad at pagkakahubad sa mga namanang ontolohiya; sumisipat din ito sa sandali ng muling pag-alam sa sarili.

Habang binabása sina Derrida, Foucault, Barthes, at lalo na ang trinidad ng tinatawag na aralíng poskolonyal na sina Said, Bhabha, at Spivak [na tagasalin ni Derrida], maaaring sabihing patuloy ding nililingon-lingon ang dalawang pangunahing pigura ng mas nauna pang pihit pa-kultura sa Filipinas na nanguna sa pagkalap ng masasabing pirasong-Filipino na kailangang mapagsama-sama upang maigiit ang ating kakayahan at kakanyahan sa harap ng pananakop.

Si Rizal ang ating walang maliw na amang pangkultura, kung nais nating maging Edipal, ang totoong aninong ating pinaglalabanan, sapagkat sa maikli niyang búhay, isinalaysay niya ang kaniyang hinagap hinggil sa bansa sa pamamagitan ng pagkalap ng kuwentong-bayan, kodipikasyon ng kauna-unahang ortograpiyang pangwika, pagpapaliwanag ng katutubong panulaan, at pagsususog sa kasaysayang unang isinawika ng banyaga para sa atin. Ang rurok ng kaniyang karera ng paglilingkod ay ang kaniyang dalawang nobela na naging dahilan ng sariling kamatayan at nagpasiklab sa himagsikan.

Ama rin natin si Delos Reyes dahil nagtangka siyang pangalagaan ang ating artsibo-mula sa mga kuwentong bayan at mga kaugalian hanggang sa mga enggrandeng katutubong ritwal at pagtatanghal. Minithi niya ang lumikha ng ensiklopedikong kaalaman hinggil sa ating kultura, at masasabing isa iyong kaunaunahang "pagsagot" o "striking back" sa imperyong minsang tinuya táyo sa ating lubhang kamangmangan at katamaran.

Taglay na nina Rizal at Delos Reyes noon pa man ang mga kritikal na katangiang ibabandera ng mga gawi ng kontemporaneong pihit pa-kultura: (1) ang pagkakosmopolitano, dahil sa pagbúbukás ng sarili sa daigdig; (2) ang pagkamausisa, sapagkat walang tigil sa paghahanap ng paliwanag sa mga bagay-bagay; at (3) ang tatawaging "pagkainterdisiplinaryo," sapagkat ang proyekto ng pag-aakda sa bansa ay isang kompleks na paghihinuhang kailangang sumandig sa bawat makatutulong na sangay ng karunungan.

Magkakaugnay ang tatlo sapagkat binibigkis nito, kung baga sa walistingting, ang lahat ng karunungan at kaparaanang kinasangkapan ng dalawang bayani sa kanilang gawain ng pag-aakda sa bansa. Kapwa pagsaklaw sa lahat ng maaaring kabatiran ang operasyonalisasyon ng tatlong ito sa kanilang kamalayan. Pangangailangan ang pagiging kosmopolitano upang makasilay sa kaugnayan ng bayan sa uniberso ng moderno. Pangangailangan din ang pagiging likás na mausisa upang masalà't masuri ang maraming ipinamálay sa atin bílang mga sakóp. 
Higit na pinakamalaking pangangailangan ang pagiging masaklaw sa mga metodo ng pag-alam na sa taóng 1937 lámang mapapangalanang paraang “interdisiplinaryo," sang-ayon sa Online Etymology Dictionary ("Interdisciplinary" np). Isang likha ng modernidad at kahingiang kosmopolitanismo ang mga pagkabatid na dala hinggil sa sarili at paligid ang pagsasamot ng mga pamamaraan ng mga disiplina, at naging kagamitan iyon ng mga edukado nating bayani tulad nina Rizal at Delos Reyes.

\section{PARA KANINO?}

Isang bandila ng pihit pa-kultura, na siya ring nagdulot dito ng kakaiba at sari-saring wika, ang interdisiplinaridad. Itong gawi ng pagsaklaw sa iba pang tradisyon ng pagalam, lalo sa mga aralíng panlipunan, ang nagpalawak at nagpatimyas sa pananaw ng aralín. Sa pagmumuni natin hinggil sa pihit pa-kultura sa Filipinas, magandang banggitin na noong mga "panahon ng sigwa"-halos sakto sa pagdako ng hanging teoretiko sa bansa-maalingawngaw ang pagtatanong hinggil sa malikhaing praktika ng "para kanino?" lalo sa ating mga nakikisangkot na manunulat.

Para kanino nga ba ang panulat? Para kanino ang paglikha? Isa itong makabayang pihit, at nakasalig sa makabansang tunguhin ng mga kilusang lumalaban sa paparating at hindi napigilang diktadurya. Naging linya ang "para kanino," naging gabay upang uriratin ng manlilikha ang ubod ng kaniyang sining. Sapagkat madalas na manlilikhang sining din ang mga kritiko, sumapi rin sa kritisismo ang diwain ang pag-uusisang ito, na sa konteksto ng diktadura ay sikil at palagiang pinupulis.

Ang tanong na ito ang naging mahalagang aspekto ng pagteteorya sa sarili ng mga kritikong nakasumpong sa panahon ng mithiing igiit ang sariling kalinangan, at ipagpatuloy, wika nga, ang mga nasimulan nina Rizal at Delos Reyes, sampu ng mga sumunod sa kanila. Sabay na muling tinangkang buwagin, kung baga, ng makabagong kaisipang teoretiko at ng "rebolusyonaryong" mithiing muling nabalikan, ang malaong mga pagtatakdang kolonyal at ang mga panibago nitong anyo.

Ang pinakarurok ng pangyayaring ito ay ang kumalat na Filipinisasyon sa buong akademya noon ding mga panahong nabanggit. Sa mga pamantasang malaong hawak ng mga banyaga (ang mga Espanyol na Dominiko, halimbawa, sa University of Santo Tomas, at ang mga Americanong Heswita sa Ateneo de Manila University), nagkaroon ng paghingi sa pamamahalang Filipino sa matataas at susing tanggapan. Ang mga pagpapalit-tao ay magdadala ng higit na katiyakan sa sarili ng mga nasa bokasyon ng paglikha, pananaliksik, at pagtuturo. Itong pagtatanong din ng "para kanino" ang masasabing nagtulak ng laksang kabaguhan sa maraming kagawaran ng 
Ingles at panitikan, na nagsimulang bumaling sa materyal na Filipino at magbukod ng sarili mula sa napaglulumaang paradigma ng kanon.

Para sa marami, isang retorikong tanong ang "para kanino" at hindi gaanong napahahalagahan sa usapin ng pihit pa-kultura. Ngunit makikita sa ating pagsusuri ngayon lalo na sa muling pagdawit kina Rizal at Delos Reyes, na napakahalaga nito sa pagsusog at pagpook sa pagdako ng teorya sa bansa. Sapagkat hindi lámang nito pinabalik ang mga palaisip sa diskurso ng paghahanap sa sarili-anuman ang anyo nito.

Sapamamagitan ng mga metodong masaklaw, ng mga metodong interdisiplinaryo, pinabalik din táyo sa mismong rasyonale ng sinasabi ko nga kaninang pagpulot, pagbubuo sa mga pirasong Filipino na sa matagal na panahon ay hindi naman tinutunghan. Sapagkat iyon naman ang tinupad ng mga exemplar sa papel na itoang pagpulot, pagbubuo sa mga pirasong Filipino.

Ang pihit pa-kultura, kung babalingan sina Rizal at Delos Reyes, ay gawain ng pag-aartsibo, anotasyon, o eksplikasyon. Nagiging makahulugan ang ganitong mga gawi paglaon, lalo sa pagsapit ng masalimuot at masigalot na panahon ng "pagdating" ng teorya sa Filipinas sapagkat sinimulan din ng mga ito ang animo'y pagpulot at pagbuo sa mga pirasong Filipino na matatagpuan sa mga kinikilalang kulturang popular o pangmasa.

\section{MGA NATATANGING TAGAPANGUNA}

Tampok na halimbawa ni Hau sa kaniyang papel ang ambag nina Reynaldo Ileto, Vicente Rafael, at Zeus Salazar sa larang ng kasaysayan; at sa aking palagay, sa liwanag ng aking usaping binuksan, ang kanilang ambag ay lumalagpas din sa mga hanggahang naipook na nga ng sumulat ng talakay.

Ramdam at pinahahalagahan sa panitikan ang kanilang pagsusuri sa kasaysayan sapagkat hindi lámang nila tinangkang bigyang-tinig ang malaong walang tinig, ang mga nása ibaba o laylayan. Gumamit sila ng mga teksto mula sa kulturang Filipino upang pag-aralan ang mga katutubong paghinuha sa sarili at daigdig at naitaguyod nilang muli ang kakaibang henyo ng nakaugat na mga halagahan.

Sa kaniyang tangkang ilahad ang kasaysayan ng rebolusyon "mula sa ibaba," binása ni Ileto ang Pasyon, isang popular na teksto, at nahango niyang muli ang mga katutubong pananaw na ginamit ng mamamayan upang "basahin" ang tekstong ito na noong una'y naglayong papayukurin ang katutubo na tulad ni Kristo. Binása 
naman ni Rafael ang mga sinaunang limbag na aklat-kolonyal upang suriin ang proseso ng mapaglarong pag-unawa ng mga katutubo bílang isang kakatwang pagsasalin. Nagtangka naman si Salazar na magtaguyod ng isang pananaw, ang "pantayong pananaw" sa pagkakasaysayan, na nagagabayan ng mga katutubong dalumat ng komunidad at pagiging bahagi nito.

Mahalagang banggitin ang pagkasangkapan ng tatlo sa popular o katutubong materyal sapagkat ganitong geist o diwain ang naitindig ng pihit pa-kulturang nakatingin man sa Kanlurang teorya ay nakatuon din sa naunang mga rebolusyonaryong mithiin. Kapara ng kanilang mga tagumpay ang naging tagumpay ng mga kritikong pampanitikan na sumalok din sa katutubong bait at popular na materyal, sa paraang pinupulot din at binubuo ang anumang matatagpuan pang pirasong Filipino.

Maitatanghal sa bahaging ito ang mga akademikong tulad ni Bienvenido Lumbera na nag-aral at nagpakilala ng malawakang panulaang Tagalog mulang tradisyong pasalita hanggang sa panahon ng rebolusyon, ni Virgilio Almario na nag-aral din ng panulaang Tagalog at nagpakilala sa atin ng tagisang Balagtasismo versus Modernismo, ni Soledad Reyes na bumaling naman sa mga popular na nobela at binulatlat ang mga ito bago pumalaot sa iba't ibang teksto ng kulturang popular, at ni Resil Mojares na nag-aral din ng nobela at intelektwal na kasaysayan ng bansa habang binabalingan din ang kaniyang ugat na Sebuwano.

Pinulot at binuo ng mga kritikong ito ang mga "pirasong Filipino" na isinantabi ng mga dakilang tradisyong ipinakilala ng Kanluran at Kanluraning edukasyon. Tinangka nilang mag-ambag ng hinuha hinggil sa mga kulturang kanilang nahango mula sa mga tekstong kanilang pinaksa. Sa pamamagitan ng kanilang mga akda, nagawa rin nilang kasangkapanin ang teorya upang lumikha ng mga bagong teorya at haka hinggil sa kulturang Filipino, na sa pangkalahatan ay ginawang bukod (other) ng kolonyal na danas at edukasyon.

Sa kanilang mga batayang artikulasyon din nailantad ang tunay na dikotomiyang umiiral at sumisiil sa kalinangan noon pa man. Madaling sabihin na kapara rin nito ang mga kategoryang High art/low art at Panitikan/panitikan, ngunit sa ganang akin, may higit itong kasalimuotan sapagkat wari'y nilalahat ang kabuuan ng kulturang Filipino bílang bukod.

Kung sa mga akda ng mga historyador na tinukoy ni Hau ay tinangkang ilantad ang "Great Divide" o yaong sinasabi ni Salazar na "Dambuhalang Pagkakahating Pangkalinangan" (54), mahihiwatigan naman sa ating mga tampok na kritiko ang paglalantad sa dakilang pagkakasantabi ng malawak na kategoryang "Kanluran," manapa, Ingles, sa katutubo, o sa isang bandá, sa "Filipino," sa lokal. Masigasig ang 
magiging artikulasyon nina Lumbera, Almario, Reyes, at Mojares hinggil sa bagay na ito-mulang mga di maglalaon ay poproblemahing esensiyalista hanggang sa masasaklaw, masasalimuot na pagdalumat. Ngunit hindi kalabisang sabihin na lahat ay pagtungo, pagbaling sa marhinalidad [halimbawa, pagkababae, pagiging mahirap, pagkakasalaysayan sa kultura] na unang sinikap ilantad ng aralín bílang pook ng potensiyal, subersiyon, at paglaya.

\section{PAGSAWATA SA BISÀ NG INGLES}

Ang talagang dakilang nilalabanan at pinupuksang sumpa sa larang na ito, kung gayon, ay ang Kanluran o Americano, na ang pinakamakapangyarihang bisà sa ating kultura ay ang wikang Ingles. Sa kasalukuyan, Ingles ang kumakatawan sa lahat ng mataas na pagpapahalagang ipinaangkop ng Kanluran upang igiit ang posisyon nito sa dinamikong pangkultura sa kasaysayang Filipino.

Ito ang maydala ng sakít na malaon na nating inihihinaing, ang sinasabing mentalidad na kolonyal, na tanging pagiging mababang uri ang naipasasaloob at naipararamdam. Ito ang monumento ng minsang inilarawan ni Renato Constantino na "miseducation of the Filipino."

Sa kabilâng banda, ito rin ang nagtulak sa ating mga iskolar noong masalimuot at masigalot na panahon ng pagdating ng Aralíng Pangkultura na itatag ang larang ng pag-aaral ng sarili sa wika, o mga wika sa bansa, na tunay na nakahuhúli sa mga inilalatag na hilig, haka, o hinuha. "Originality as vengeance" ang minsang ibinansag dito ni Lucilla Hosillos, na isa pang mahalagang tagapag-ambag ng kaisipan sa ating pihit pa-kultura. Ang "pagganti" —sa Kanluran o sa Ingles—ay matatagpuan sa pinakasalitang ugat ng "originality" na "origin," sa minulan at sinapupunan. Isa itong pangangailangang pagbabalik, at sa pangmalawakan, pagliligtas sa lahat ng mga naibukod ng Kanluran/Americano/Ingles.

Minamalas ko itong dalawahang kategoryang ito at hindi ko maiwasang isipin kung papaanong sa panahon pa lámang nina Rizal at Delos Reyes ay sinasawata na ito. Naroroon na ito nang mapang-uyam na tinugunan ni Rizal, halimbawa, ang sinasabi ni Vicente Barrantes na "ineptness and penchant for lower-order mimicry" ng komedya ng mga indio (Mojares 186).

Naroroon din ito sa sariling pagtatanggol ni Delos Reyes sa komedya na kaniyang itatanghal bílang isang dramang "indigenized and exhibits artistic values comparable with the great literatures of the world" at "a traveling hybrid form that can be claimed as 'our own"' (Mojares 188). 
Sa mga pagsusuri sa pagtatanggol nina Rizal at Delos Reyes na sinipi natin mula kay Mojares, mahihiwatigang mistulang nauna pang di hamak nang isang siglo kay Bhaba ang bayani-intelektwal na Ilokano na makahinagap ng pagkahalo-halo o hybridity ng ating kultura.

Sa hulí, nahinagap nila ang mga iyan dahil nása mata mismo sila ng maunos na salungatang Kanluran/lahat ng katutubo, pinapasò sa kanilang maaanghang na sagot ang mga mangmang na nagdudunung-dunungan. Marahil, ganito ring mata ng unos ang tinunghan ng mga iskolar at kritiko nang itulak sila ng historikong pagkakataon at ng pagpasok ng Aralíng Pangkultura sa larang sa pakikisangkot.

Sa mga panahong ganap nang nahalinhinan ang Kanluraning institusyon ng aparato ng Ingles na "buong pagmamalasakit" na inihandog sa atin ng mga Americano, nagkaroon ng muling pagkamulat mula sa mga pagkakadusta; halimbawa, mula sa pagkakalugar sa panitikan sa Filipinas bílang "inchoate," walang lawas na maipagmamalaki.

Ang ganitong malawakang pagmamaliit sa kulturang Filipino sa pangkabuuan ang masasabing dahilan ng pagpihit ng kilusang Filipinisasyon sa mga materyal na Filipinong hindi makatatanggap ng kanonisasyon, kahit kailan, mula sa makaIngles at nag-i-Ingles na akademya.

Ang pagsibol, halimbawa, ng mga pagpapahalaga sa mga panitikang nasusulat sa wikang rehiyonal (bukod sa wikang pambansa na minamaliit din), popular na panitikan, pagtatanghal at praktikang pangkultura, at pati na mga papausbong na midya, lalo na ang pelikula, ang magiging paksa ng mga pag-aaral. Lahat ng mga ito ay pawang hindi nasusulat sa Ingles, at madalas itampok sa halagahang "tatlong B," sa kritika ni Reyes: "bakya, baduy, at basura" (325).

\section{SAMUTSARING USAPIN}

Sa aking obserbasyon, palaging nilalagpasan ang usapin ng wika sa pagtukoy sa mga tekstong ito na "bakya, baduy, at basura." Wari bang itong pagbubukod sa mga ito ay pamana lámang ng teorya sa atin, lalo’t matapos matutuhan ang pagkakabuwag sa, at patuloy na problematisasyon ng High art/low art.

Bagaman mayroon ding "giriang" maiuugnay sa nasabing halagahan sa panitikang Ingles sa Filipinas-magugunita ang "art for art's sake" ni Jose Garcia Villa at ang lumalaban ditong "literature and society" ni Salvador P. Lopez noong panahon ng Americano-ibang sapin ng pagkamasalimuot ang naidudulot ng pagbalik sa ating 
gunita ng pagkakasantabi ng mga pangkulturang manipestasyon ng lokal na wika/ mga wika.

Kapwa Ingles ang gamit nina Villa at Lopez sa kanilang tagisan at ang pinagtatalunan nila ay ang mismong pinakawastong politika. Sinuman ang makapangibabaw, kapwa sila maiaangkop sa larang sapagkat, una, sumusulat sila sa mga anyong pampanitikan na pinasibol at pinapaghusay sa kanila noong panahong Americano, at ikalawa, ginagamit nila ang banyagang wika na may bisà ng lehitimasyon.

Bagaman hindi naman natin maitatatwa ang pagkamakabayan ni Lopez at ng kaniyang mga kasapakat, itong Ingles na ginamit nila ang nagtatag sa kanila bílang mga lehitimong institusyon sa larang—na sa kasawiampalad, nagpabukod sa mga dapat na maibukod. Malalim itong ganitong mentalidad na maka-Kanluran/makaAmericano/maka-Ingles, at nakikita ko pa ito sa sarili kong kritika ng teleserye.

Madalas na hinaing ng mga manonood, halimbawa, ang wari nila'y kawalan ng pagkamalikhain sa mga teleserye, ang pagiging gasgas ng mga kuwento nito. Ngunit sa tuwing durugtungan nila ang kanilang hinaing ng "kung sana'y matutulad lámang ang mga ito sa mga seryeng gáling America..." talagang hindi mahirap magunita kung gaano kabisa ang gahum ng nasabi nang mentalidad.

May prehuwisyo na kaagad sa mga tekstong katulad ng teleserye sapagkat malalim nga ang pagkababad sa gayong kaisipang nagtatalaga sa Kanluran o Ingles bílang superyor na minulan ng lahat. At hindi lámang dahil talagang mababa ang tingin sa anyo, sa kasong ito, ng teleserye, halimbawa.

Sa kabila ng pagkilala at pagtangkilik dito ng iba't ibang bansa, at matalinong pag-angkop nito sa mga impluwensiyang banyaga upang makasunod sa agos ng global na merkado ng drama, hindi pa rin ito pinapansin, at kung pinapansin man, patuloy pa ring pinagdududahan at iniismiran. Ito, sa tingin ko, ay dahil sa ang wika nito ay ang pambansang wika, ang wika ng marami.

Ang wika ng marami ay itinuturing lámang na wikang pang-araw-araw at pangkalye. Anumang nasasalat ng wikang ito ay agad na kailangang tatakang "bakya, baduy, at basura," at kahit pa magsumikap ito na kamtin ang lahat ng uri ng kasiningan ay hindi pa rin makaaabot sa antas ng Hollywood. Hindi iilang ulit akong napaaway sa social media sa pagtatangkang iwasto ang ganitong kaisipan, ngunit sa totoo lámang, mapasusuko ang sinumang maglalakas-loob sa lubhang napakalalim at nagkalat na kamangmangan. 
Sa kabila nito, maraming matututuhan sa naging tugon ng ating mga kritiko sa pagsawata sa ating ipinanukalang bagong pag-unawa sa dikotomiyang pangkultura. Makikitang buháy ang pagkamálay ng mga kritikong Filipino sa ganitong tagisan, sa isang mithiing makipagtalaban at makipagsabayan sa tagapagtatag ng gahum.

Isa na riyan si Lumbera na sa sanaysay niyang "Popular Culture as Politics" ay nagpahalaga sa subersibong potensiyal ng kulturang popular, bagaman ito'y tinukoy na "cultural forms and their respective content, which have been introduced from without, before these have been assimilated into the sensibility and values system of the people" (155).

Ginawa niyang halimbawa ang mga pamanang Kanluran na komedya [na binanggit na nina Rizal at Delos Reyes!], ang nobela, at ang mga modernong anyong pang-aliw bílang mga anyong makapangyarihan na nakasangkapan ng katutubo upang sagutín ang mga panunudyo sa kaniya. Dagdag pa niya: "Popular culture is power, and whoever wields it to manipulate minds is likely to find its literary and technological machinery turned against him when the minds it has manipulated discover its potency as a political weapon" (160).

May ganito ring "pagliligtas" sa kulturang popular si Isagani Cruz. Sa kaniyang sanaysay na "Lakas ng Libro/Lakas ng Tao: Pagdikonstrak sa Texto ng Pebrero," kakasangkapanin niya ang natutuhan sa pos-estrukturalismo ["istrakturalismo" sa kaniyang tinuran] upang igiit na ang kultura, tulad ng panitikan, ay makahulugan at maaaring matalik na mabása. Inspirado ng paglalaro sa wika ni Derrida, itinanghal niya rin ang kakayahan ng wikang Filipino sa kritisismo at pagdalumat sa pamamagitan ng pagpapakilala sa terminong "texto" (kabukod ng "teksto"), o yaong "hindi masasabing tuwirang pangliteratura, ngunit mahahanapan pa rin ng katangiang tinatawag ng mga Formalistang Ruso (Russian Formalist) na literariness" (52).

Sa isa pang sanaysay, ang "Katitikan, Panitikan, Literatura, at Panulat: Isang Panimulang Hakbang Tungo sa Isang Maka-Filipinong Teoryang Pampanitikan," higit na lilinawin ni Cruz ang saklaw ng sinasabi niyang "texto": "Dapat sigurong banggitin na hindi makitid ang aking pagbibigay-kahulugan sa salitang "texto." Isinasama ko rito ang mga mala-literaturang likha tulad ng mga palabas sa telebisyon at mga pelikula" [akin ang diin] (90).

Sa ating panukalang pag-unawa, ang paglalarawan sa mga teksto bílang "malaliteratura" ay maparikala at isang mapanuyang tugon sa establisimiyentong mapanggahum. Masasabing pagkadulas din ng dila ito, na pagtanggap nga sa mga naunang pagtataya sa mga tekstong ito. 
Ngunit isang mahalagang pihit pa-wika ang tinupad ni Cruz, sampu ng iba pang ipinakilala niyang termino na sumasalok sa bait ng mga katutubong wika, kabílang na ang paggamit sa salitâng gahum, mula sa Visayas, para ipalit o ipansalin sa hegemony ni Antonio Gramsci.

Hindi simpleng pagsasalin ang tinutupad ni Cruz sa mga terminong nasalok sa teorya-pagtugon ito sa pamamagitan ng paggigiit sa kritikal na kakayahan ng wika, na bílang diskurso ay kasámang minamaliit ng lahat ng kabukod ng panig ng Kanluran/Americano/Ingles. Nakatulong ang pananaw ni Derrida upang yanigin ang kolonyal na kamalayan sa namamayaning kritisismong nakapailalim sa rehimen ng aklat at pormalismo.

Halos kapara rin ng operasyong ipinatupad ni Cruz sa kaniyang kritika ang ginawa ni Rolando Tolentino sa kaniyang "mapagpalayang" pagbása sa iba't ibang teksto-mula sa panitikan hanggang sa kultura ng mall at ng mga celebrity sa telebisyon at pinilakang tábing.

Para sa akin, pinakatampok sa pag-usig sa maka-Kanlurang paradigma ang mga naging interbensiyon ni Reyes. Sa kaniyang sanaysay na "Ang Mambabasa/ Manonood, ang "Mass Media" at ang Paglikha ng Kahulugan," inililigtas niya mula sa kinasasadlakang pagkakasantabi ang kulturang popular sa pamamagitan ng pagliligtas din sa malaong tinatalikuran sa pag-aaral ng mga panitikan: ang mambábása.

Ang pagpihit patungo sa mambábása ay tunay ngang pihit pa-kultura sapagkat sino pa nga ba ang bumubuo rito kung hindi ang mga táong tumatanggap at nakikibahagi sa pagpapakahulugan nito? Ang mga tumatanggap ang siyang kultura, at ipinamamalay na muli sa atin ito ni Reyes. Sa kaniyang kritisismong hubog din ng teorya, lalo na ng teorya ng pagtanggap o resepsiyon, napagitaw niya ang malaong pagtalikod sa tagatanggap ng pampanitikang mensahe dahil na rin sa pagkiling ng ating edukasyon sa akda/teksto bílang tanging salalayan ng danas pampanitikan at kahulugan.

Sa isa pang sanaysay, ang "Ang Puno't Dulo ng Kulturang Popular," tutukuyin niya ang implikasyon ng binanggit na kaninang "tatlong B." Wika niya, ang pagtatalaga sa kulturang popular bílang ang "tatlong B" ay pagtatalaga rin sa mambábása/ manonood sa kawalang-ahensiya at kakayahan sa pagsuri sa anumang tinatanggap.

Sa “Ang Pagbasa Bilang Paglikha," binigyang-diin ni Reyes na sa tradisyong pampanitikan ng Filipinas, malinaw sa mga manunulat ang mahalagang papel ng mambabása (25) at sa gayon ay binasag niya ang masyadong pagkiling sa pormalismo. 
Makikita raw ito sa nakagawiang "pag-uukol ng ilang pananalita na inihahandog sa Bayang Maralita/Sambayanang Pilipino/Kapwa Pilipino" ng mga batikang nobelistang tulad nina Francisco Laksamana, Lope K. Santos, Iñigo Ed. Regalado, at Juan Arciwals, atbp., bukod sa malinaw na katalagahan sa tungkulin ng nobela bílang genreng "bukal ng mga aral na maaaring pagbungahin sa buhay ng mga mambabasa" (25).

Ganitong mapagbagong balangkas ang pinaniniwalaan niyang makapaghahango sa kulturang popular, na inilarawan niya sa "Puno't Dulo ng Kulturang Popular" na mga "kontemporanyong ekspresyon ng mga pananaw na matatagpuan sa kapaligiran" (323). Ituring mang "ilehitimong ekspresyon ng kultura," iginiit ni Reyes na "(w)alang maituturing na pagkakaiba ang Kultura para sa iilan at ang Kultura para sa nakararami” (324). Bukod sa ito ang namamayaning "kultura ng nakararaming mamamayan, pagsasakatawan din ito ng "mga pangarap, ideya, at saloobin ng nakararami" (324).

Tulad ng kaniyang mga kapanahong kritiko, nakita niya ring makahulugan ang kulturang popular, at kung matutunghan lámang ay katatagpuan ng mga sumusunod: (1) "masasalimuot na realidad na naglalaman ng mga karanasan at damdaming ipinamamalas sa pamamagitan ng iba't ibang anyo"; (2) mga "signipikasyon (at maging) mga tangkang maunawaan ang masalimuot na karanasan at pangyayari sa loob at labas ng buhay at indibidwal at isang lipunan"; (3) mga paraan ng pagunawa sa pamamagitan ng "mekanismo upang kayanin ng tao na batahin ang komplikadong buhay"; (4) mga "tuwirang aral at leksiyon sa buhay," na animo'y "makapangyarihang tradisyon sa ating panitikan o pelikula"; at (5) aliw at ligaya sa pagdalaw sumandali sa "ibang daigdig" upang takasan "ang ordinaryo at kabagutbagot na tunay na mundo" (327-329).

Kapag binása natin ang mga tinuran ni Reyes hinggil sa potensiyal ng kulturang popular, hindi maiiwasang ihambing ito sa mga sinabi na ng mga nanguna sa larang na ito sa Kanluran:

Sa madaling salita, maraming bagay na dapat punahin sa larangan ng kulturang popular. Subalit gaya nang naipakita na, marami ring bagay at katangiang taglay nito na dapat masusing mapag-aralan sapagkat bunga ang mga ito ng masasalimuot na proseso ng paglikha ng kahulugan ng ating mga mamamayan. Samakatwid, bahagi sila ng proseso kung paano nabubuo ang partikular na kamalayan ng isang indibidwal sa loob ng isang sistemang ang mga klase at uri ay may pagkakaiba dahil sa pagkakaroon o kawalan ng kapangyarihan (342).

Subalit higit itong nagkakaroon ng partikular na bisà kapag minamalas gámit ang ating mungkahing balangkas kung saan ang dikotomiyang talagang kailangang 
baklasin sa ating diskurso ay ang Kanluran-Ingles/ang kabuuan ng kulturang Filipino. Una'y nalilinawan táyo na hindi lámang ito usapin ng Mataas na Sining/ mababang sining na sumasaklaw sa makauring pagtatakda ng panlasang palagiang nagtuturing sa kulturang popular sa pook na marhinal.

Dapat lámang itong pagtaasan ng kilay sapagkat di tulad ng Mataas na Sining, na may kaisahan at kumakatawan sa mabuti, totoo, at maganda, ang mababang sining ay nagtataglay ng "maraming bagay na dapat punahin." Bahagi rin naman itong pananaw na ito ng ating konteksto ng pagkamálay sa kulturang popular. Ngunit may taglay pang mas mabigat na "bagahe" ang Filipinong kulturang popular, at tinunghayan na nga natin iyan sa kabuuan ng papel.

Higit na nagpapabigat sa usapin ang pangmatagalang epekto ng Ingles at edukasyong Americano sa ating kamalayan. Kayâ't kapag tinitigan natin ang sinasabi ni Reyes na "masasalimuot na proseso ng paglikha ng kahulugan ng ating mga mamamayan," makikitang nakaugat dito ang salimuot ng ating nagpapatuloy na pagbubuo ng sarili bílang isang bayang dáting sakóp at kasalukuyang binabatá ang "isang sistemang ang mga klase at uri ay may pagkakaiba dahil sa pagkakaroon o kawalan ng kapangyarihan."

Sari-saring sapin ng "pagkakaiba" ang maaari nating matawag sa usaping ito, bukod sa panlipunang pagkakaiba na ugat ng kawalang-pagkakapantay. Naririyan ang ugnayang sentro-laylayan na lalaging multo sa mithiing papag-isahin ang bansang pulo-pulo. Naririyan din ang umiiral na tagisang Pambansang Wika/ Wikang Rehiyonal na nagpapatindi sa hámon ng pagkakaisa. At gaya ng ating nabanggit, naroroon ding kasangkot ang kabuuan ng mga wika sa Filipinas na nasasangkot sa pagtatanggol ng sarili sa palagiang "anino" ng Ingles.

Ang ating multikulturalidad ay hámon din sa ganitong pagbubuo, bagaman tinitingnan ito madalas na isang pook ng laksang posibilidad ng integrasyon. Hindi maitatangging lahat ng ito ay kinapapalooban ng usapin hinggil sa "pagkakaroon o kawalan ng kapangyarihan." Namumuhay sa ganitong sapin-saping kontradiksiyon ang Filipino, at kailangan niya itong yakapin bílang paraan ng paghinuha niya sa sarili. Bahagi ito ng kaniyang "proseso ng paglikha ng kahulugan" at pagbubuo rin ng kaniyang kamalayan, bílang isang indibidwal at bílang bahagi ng kolektibong Filipino. Mahirap maipirme itong kamalayang ito kung kayâ't nararapat lámang na "masusing mapag-aralan" sa pamamagitan ng mga tekstong nabanggit. 


\section{ILANG TUON SA ARALÍNG TELESERYE AT PAGLALAGOM}

Sa pangkalahatan, pinahahalagahan ni Reyes ang konteksto at ang matalik na kaugnayan nito sa kulturang popular. Sa aking pagsuong sa masasabing madilim, mapanglaw pang kagubatan ng aralíng teleserye, dala-dala ko ang kislap-diwang ito, lalo pa't nahaharap ako sa hámon ng paggigiit sa dalawang bagay-na (1) isa ngang mahalagang teksto ng ating panahon ang teleserye, at (2) kailangan itong mapag-aralan, agad-agad, sa láyong higit na mapatalim ang paghinuha rito bílang teksto, at sa buong kulturang popular sa pangkalahatan.

Minamasdan kong muli ang isa sa tinatawag na "learning outcomes" ng kursong aking itinuturo hinggil dito sa Ateneo de Manila University, at nahihindik ako, muli at muli, sa mapanghamong trabaho ng pagsinop sa soap opera sa Filipinas, na totoo namang hindi gaanong napagtuunan ng pansin, marahil dahil sa pangkalahatan, palaging abalá ang iskolar ng kulturang popular sa pagtatanggol ng kaniyang paksa at pag-iral, sa isang akademyang humaharap sa mas malakihang suliranin ng misedukasyon sa panitikan na laganap sa sistema ng batayang edukasyon: "Defend the relevance of studying teleseryes not only as a means to make sense of current affairs and culture, but also as an important contemporary form of popular literature that cuts across social classes, as well as national and transnational borders."

Papaano nga ba magaganap ang anumang "pagtatanggol" sa teksto kung ang karaniwang mga teksto naman ay ni hindi malapitan nang mabuti? Papaano ipapamálay ang matataginting na pagbása kung salat naman sa kaalaman? Isa pang sapin ng balintuna itong binanggit ko, na maaaring lalong magpasalimuot sa patuloy na kinakabaka ng madla na "pagkakaroon o kawalan ng kapangyarihan."

Ang pagkamangmang ay isa ring pagkakalugar sa isang marhinal na posisyon, at makatwiran namang isipin na kailangan munang "mailigtas" ang madla mula rito, bago pa man táyo makapaghayin ng higit na masasalimuot na paghihinuha sa masalimuot nang búhay. Maraming maaaring maging paliwanag sa kakulangan sa iskolarsyip na aking binabanggit [bagaman, sa larang ng pananaliksik sa komunikasyon o pangmadlang komunikasyon, halimbawa, búhay ang masasabing mga pag-aaral hinggil sa manonood, at palagiang pinag-aaralan ang palagay ng mga tagasubaybay ng mga teleserye; interesante rin para sa pag-aaral kong ito ang ginagawa sa larang na iyon, bagaman nakukulangan ako sa "tekstuwalisasyon" sa kanilang mga datos o metodong madalas ay nagtuturo lámang sa mag-aaral, halimbawa, na gumamit ng mga ito para sa "market research," at hindi para sa mas malalalim na mga pangkulturang pagninilay].

Isa na riyan ang maaaring patuloy na kababaang tingin sa teleserye, dahil marahil sa pagiging malaganap at pang-araw-araw nito [iyon nga ang dahilan kung bakit ito 
kinailangang pag-aralan!]. Sa isang bandá, itong diwaing ito ng palaging pagpopook sa konteksto ang sa tingin ko'y pinakamahalagang kaisipan, gaano man ito kapayak, na natutuhan natin sa "tradisyong" ito ng pag-iisip hinggil sa lahat ng katitikanmaging Panitikan man ito o kulturang popular. Ang tradisyon natin ay masasabing isang masalimuot na tradisyon ng paghinuha. Hubog ito ng banyaga, panday ng kasaysayan ng pagkakasákop, ngunit lalaging "lumilingon sa pinanggalingan." Maligaw man, tiyak na makaiisip táyo ng mga paraan pabalik dahil araw-araw, binábása at binubuo natin ang ating sarili. Hindi táyo basta-basta mamamatiyanak, saanman táyo mapapihit. 


\section{Mga Akdang Binanggit}

Baker, Chris. Cultural Studies: Theory and Practice. Sage, 2000.

Cruz, Isagani. The Alfredo E. Litiatco Lectures of Isagani R. Cruz. Edited by David Jonathan Y. Bayot. De La Salle UP, 1996.

Hau, Caroline. "The 'Cultural' and 'Linguistic' Turns in Philippine Scholarship." Ruptures and Departures: Language and Culture in Southeast Asia, edited by Corazon Villarreal, Lily Rose Tope, at Patricia May B. Jurilla, Department of English and Comparative Literature, U of the Philippines-Diliman, 2002, pp. 36-70.

Hosillos, Lucilla. Originality as Vengeance in Philippine Literature. New Day, 1984.

Lumbera, Bienvenido. Revaluation: Essays on Philippine Literature, Cinema \& Popular Culture. U of Santo Tomas Publishing House, 1997.

Mojares, Resil. Isabelo's Archive. Anvil, 2013.

Reyes, Soledad. Pagbasa sa Panitikang at Kulturang Popular: Piling Sanaysay 1976-1996. Ateneo de Manila UP, 1997.

Rivkin, Julie, and Ryan Michael, editors. Literary Theory: An Anthology. Blackwell, 2004. 